Calculations are given for reactor parameters, and it is found that the power required to drive the current is approximately $3 \%$ of the total fusion power output and that only $1 \%$ of the area of the wall would be required for the wave guides.

\section{Masses of exotic nuclei}

\section{from John Edgington}

THE stable nuclei occupy a narrow strip on a plot of proton number $\mathrm{Z}$ versus neutron number $N$. Increase $Z$ or $\mathrm{N}$ by a few units, and the resulting nuclei undergo $\beta$-decay; increase $\mathrm{Z}$ or $N$ greatly and even this limited stability disappears, the excess nucleons being ejected singly or in clusters.

This fragility of all but a limited number of nuclei has to be explained, eventually, in terms of the individual nucleon-nucleon interactions. Given the complexity of many-body calculations, it is hardly surprising that for practical purposes, such as estimating nuclear binding energies and masses, simpler prescriptions are used. These 'semi-empirical' formulae are based on the liquid-drop model but include the main features of the individual interactions in an approximate way. The values of the numerical coefficients involved are adjusted to fit the masses of stable nuclei, and the most widely used of these formulae, the GarveyKelson relation (Garvey et al., Rev. Mod. Phys. 41, Sl; 1969) also considers correlations between nuclei with closely similar values of $\mathbf{Z}$ and $\mathrm{N}$.

The most stringent tests of these formulae occur, of course, for nuclei far from the narrow stability strip. Since these are very short-lived, some subtle experimental techniques are needed. A fine example is that reported from the LAMPF accelerator, Los Alamos, by a LASL/Northwestern University group (Seth et al., Phys. Rev. Lett. 41, 1589; 1978) who have measured the mass of ${ }^{18} \mathrm{C}$. This exotic nuclide, with twice as many neutrons as protons is one of a class of nuclei having $\mathrm{N}=\mathrm{Z}+6$ which seem to mark a significant boundary on the stability map of light nuclei. If $\mathrm{N}$ and $\mathrm{Z}$ are both odd, these nuclei are unstable to particle emission; if even, they appear to be stable. Like other neutron-rich nuclei, ${ }^{18} \mathrm{C}$ can be produced fairly readily in heavy ion collisions, or as a spallation product of heavy nuclei, but these violent processes are quite unsuited to precise mass measurements.

Seth and his colleagues have produced ${ }^{18} \mathrm{C}$ by the double charge

John Edgington is in the Department of Physics, Queen Mary College, London.
Clearly an experimental demonstration of both the fast ion and r.f. schemes in a tokamak is required before any realistic assessment of the reactor potential of either of these schemes can be made. exchange (DCX) reaction ${ }^{18} \mathrm{O}\left(\pi^{-}, \pi^{+}\right)^{18} \mathrm{C}$, using the energetic pion spectrometer at LAMPF and measuring the energy of both the incident $\pi^{-}$and the outgoing $\pi^{+}$, to obtain the binding energy of ${ }^{1 \times} \mathrm{C}$ by subtraction. The technical difficulties are great. There is a huge background of electrons from $\pi^{\circ}$ production and decay, but these are entirely eliminated by time-of-flight and Cerenkov counter techniques; also, the spectrometer's energy resolution is too poor for mass measurements, so a calibration reaction involving nuclei with known masses, ${ }^{12} \mathrm{C}\left(\pi^{-}, \pi^{+}\right)^{12} \mathrm{Be}$, was also measured. The group had previously confirmed (LA-7457-PR Progress Report, Sept. 1978) that their equipment had the desired sensitivity, using the simpler reaction ${ }^{9} \mathrm{Be}\left(\pi^{+}, \pi^{-}\right)^{9} \mathrm{C}$, since a target of natural beryllium metal poses fewer difficulties than the enriched ice target used for the ${ }^{18} \mathrm{O}$ DCX reaction.

Seth et al. compared their result for the mass excess of ${ }^{18} \mathrm{C}, 24.91 \pm 0.15$ $\mathrm{MeV}$, with the Garvey-Kelson relation. Earlier predictions had suggested a somewhat lower binding energy, but a recalculation of the G-K prediction, using recent measurements of the ${ }^{17} \mathrm{C}$ and ${ }^{19} \mathrm{~N}$, yielded a mass excess of $25.04 \pm 0.10 \mathrm{MeV}$, in excellent agreement.

It is worth stressing that Seth et al.'s measurement would not have been thought possible, even with technical ingenuity, two years ago when the cross sections for the DCX reactions $\left(\pi^{-}, \pi^{+}\right)$were thought to be exceedingly small. Conventional wisdom had it that reactions connecting analogue nuclear states were much more likely than those between non-analogue states. Roughly, analogue states are corresponding states in different nuclei which differ only in that one or more protons and neutrons have been interchanged, but no rearrangement of the nucleon orbitals has occurred. According to this opinion, any necessary orbital rearrangement would depress the cross section greatly. In 1977 another group working at LAMPF, B. M. Preedom's LASL/University of South Carolina/ ANL collaboration, showed that the reaction ${ }^{18} \mathrm{O}\left(\pi^{+}, \pi^{-}\right)^{18} \mathrm{Ne}$, connecting analogue states in the two nuclei, had a cross section only about twice as large as that of ${ }^{16} \mathrm{O}\left(\pi^{+}, \pi-\right)^{16} \mathrm{Ne}$. The latter converts neutrons in a filled shell to protons occupying the next higher orbital, and so connects non-analogue states. Now, whereas it is always possible to find nuclei with excess neutrons on which the $\left(\pi^{+}, \pi^{-}\right)$reaction can connect analogue states with no orbital rearrangement, there are no stable nuclei (other than ${ }^{1} \mathrm{H}$ and ${ }^{3} \mathrm{He}$ ) with excess protons; and so $\left(\pi^{-}, \pi^{+}\right)$ reactions necessarily connect nonanalogue states.

The results of Preedom's group (Phys. Rev. Lett. 38, 149; 1977; Phys. Lett. 69A, 55; 1977) greatly encouraged study of $\left(\pi^{-}, \pi^{+}\right)$reactions, since there is now seen to be no reason to suppose that their cross sections are unusally small. Even so, Seth et al. observed only about twenty events leading to the ground state of ${ }^{18} \mathrm{C}$. They now plan to measure the mass of several more extremely neutron-rich nuclei, including ${ }^{48} \mathrm{Ar}$ which has $\mathrm{Z}=$ $18, N=30$; this will be reached by the reaction ${ }^{48} \mathrm{Ca}\left(\pi^{-}, \pi^{+}\right)^{48} \mathrm{Ar}$. We may expect other such measurements in the near future, especially as the reevaluated Garvey-Kelson relation now predicts greater stability against particle emission than before.

\section{The current state of eukaryotic messenger RNA}

from $H . R . V$. Arnstein

THE isolation of different messenger RNAs and their translation into specific proteins in a number of cellfree systems are now almost routine. Moreover, the nucleotide sequences of several messengers have been established. Given that the concept of mRNA emerged less than 20 years ago impressive progress has been made.

At a recent EMBO Workshop* the main topics were heterogeneous nuclear RNA (hnRNA) processing, hnRNAprotein complexes, free cytoplasmic messenger ribonucleoproteins (mRNPs), the cytoskeleton in relation to protein biosynthesis, polysomal mRNA-protein complexes and translational control.

The general picture which emerged was that the molecular aspects of transcription and the subsequent processing of the primary transcripts are known in outline, but there is still considerable scope for detailed studies of the enzymes involved and of the control of mRNA synthesis in eukaryotes. Moreover, almost nothing is

*Held at Hapert, The Netherlands on 27 November-1 December, 1978. The meeting was organised by $\mathrm{H}$. Bloemendal and $\mathrm{W}$. J. van Ventooij of the Biochemistry Deparement, University of Nijmegen. 